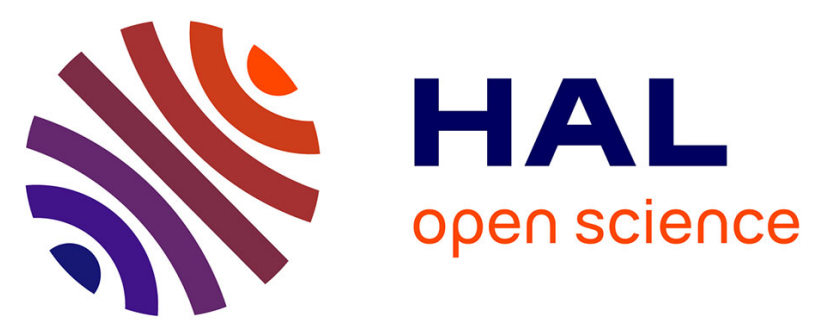

\title{
Dark-level trapping, lateral confinement, and built-in electric field contributions to the carrier dynamics in c-plane GaN/AIN quantum dots emitting in the UV range
}

Maryna Hrytsaienko, Mathieu Gallart, Marc Ziegler, Olivier Cregut, S. Tamariz, R. Butte, N. Grandjean, Bernd Honerlage, Pierre Gilliot

\section{To cite this version:}

Maryna Hrytsaienko, Mathieu Gallart, Marc Ziegler, Olivier Cregut, S. Tamariz, et al.. Dark-level trapping, lateral confinement, and built-in electric field contributions to the carrier dynamics in cplane GaN/AlN quantum dots emitting in the UV range. Journal of Applied Physics, 2021, 129 (5), 10.1063/5.0038733 . hal-03417903

\section{HAL Id: hal-03417903 \\ https://hal.science/hal-03417903}

Submitted on 29 Nov 2021

HAL is a multi-disciplinary open access archive for the deposit and dissemination of scientific research documents, whether they are published or not. The documents may come from teaching and research institutions in France or abroad, or from public or private research centers.
L'archive ouverte pluridisciplinaire HAL, est destinée au dépôt et à la diffusion de documents scientifiques de niveau recherche, publiés ou non, émanant des établissements d'enseignement et de recherche français ou étrangers, des laboratoires publics ou privés. 


\title{
Dark-level trapping, lateral confinement and built-in electric field contributions to the carrier dynamics in $c$-plane GaN/AIN quantum dots emitting above the GaN bandgap
}

\author{
M. Hrytsaienko, M. Gallart,* M. Ziegler, and O. Crégut \\ Institut de physique et chimie des matériaux de Strasbourg, \\ UMR 7504 CNRS-université de Strasbourg, 23 rue du Loss, BP 43, F-67034 Strasbourg, France \\ S. Tamariz, R. Butté, and N. Grandjean \\ Institute of Physics, Ecole Polytechnique Fédérale de Lausanne, \\ EPFL, CH-1015 Lausanne, Switzerland
}

B. Hönerlage and P. Gilliot

Institut de physique et chimie des matériaux de Strasbourg, UMR 7504 CNRS-université de Strasbourg, 23 rue du Loess, B.P. 43, F-67034 Strasbourg, France

(Dated: November 9, 2020)

\begin{abstract}
c-plane GaN/AlN quantum dots (QDs) are promising zero-dimensional quantum nanostructures that exhibit single photon emission properties up to room temperature and even above. In this context, it is of prime interest to gain a deeper insight into the recombination dynamics of photogenerated electron-hole pairs captured by such dots. Hence, in this work, we study the time-resolved photoluminescence (PL) properties in the low injection regime and at cryogenic temperatures of $c$-plane GaN/AlN QD ensembles emitting above the bulk GaN bandgap in order to properly understand the nature of the recombination channels behind the observed non-exponential decay time profiles. Such decays reveal the existence of a relaxation channel competing with the radiative recombination one. It is thus observed that for the former process the dynamics is independent of the dot height, which is attributed to a reversible nonradiative transfer that could be mediated by a spin-flip process to a dark-level state. The radiative recombination process is recognizable thanks to the characteristic dependence of its lifetime with the emission energy, which is well accounted for by the built-in electric field inherent to quantum nanostructures grown along the $c$-axis and the variations in the lateral confinement at play in such QDs. Those conclusions are drawn from the analysis of the time-evolution of the PL spectra by means of a simple analytical model that enables to exclude any screening of the built-in electric field.
\end{abstract}

\section{INTRODUCTION}

Beyond fundamental aspects, the ongoing interest in semiconductor quantum dots (QDs) is motivated by their potential for a wide range of applications such as sources of single and entangled photons [1-3], quantum cryptography [4] or spintronics [5]. For a review see for instance [6]. Key conditions to use QDs for nanophotonic applications rely on the full understanding and, if possible, the control of the different dynamics associated with electronic excitations, namely population, spin- and phase relaxation. For example, generating indistinguishable photons from single QDs requires to have a short excitonic coherence time $T_{2}$ and a short radiative lifetime $T_{1}$, which is favored in the Purcell regime $[7,8]$.

Among the wide range of available QD systems, $\mathrm{GaN} / \mathrm{Al}(\mathrm{Ga}) \mathrm{N}$ QDs offer attractive features because of the large band offsets at the AlN/GaN interface, enabling three-dimensional confinement of electron-hole (eh) pairs up to room temperature [9]. Advantage was taken of this to achieve single UV-photon emission at $300 \mathrm{~K}[10,11]$ and even above [12]. Long-lived optical ori-

*mathieu.gallart@ipcms.unistra.fr entation was also experimentally observed [13]. Such experimental accomplishments go hand in hand with strong efforts regarding QD growth in order to control their structural parameters, especially their size and surface density $[14,15]$. A fundamental and intrinsic feature of wurtzite GaN/AlN quantum nanostructures remains the

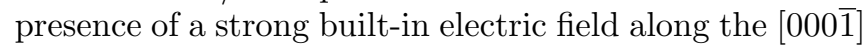
growth direction. This electric field is caused by the difference in both spontaneous and piezoelectric polarizations between the GaN and AlN materials [16], which can reach values of several $\mathrm{MV} / \mathrm{cm}[17,18]$. The presence of such a static electric field gives rise to the quantum confined Stark effect (QCSE), which has a strong influence on both the recombination energy and the lifetime of the e-h pairs. By inducing a spatial separation of electron and hole wave-functions, i.e., the formation of a dipole, the electric field decreases the oscillator strength, hence leading to a strong dependence of the radiative lifetime on the dot height $[17,19,20]$ that spans several orders of magnitude from the picosecond to the microsecond range. The consequences are dramatic for the largest QDs. First, as their radiative recombination probability is decreased, other dephasing mechanisms become dominant and the radiative lifetime of those QDs will exceed the most optimistic values of the dephasing time that can be expected for this type of system. Furthermore, their 
weak oscillator strength will hinder any resonant excitation and the control of coherent phenomena. Finally, the possible transient screening of the electric field is responsible for a complex recombination dynamics, which is non exponential and strongly depends on the number of injected e-h pairs [17]. These are major drawbacks if one considers for instance the possibility of generating and manipulating entangled states. This is what motivates the present work on small GaN/AlN QDs whose emission energy lies above the strained bulk GaN bandgap and for which a significant value of the oscillator strength is preserved. Because of their short radiative lifetime -in the nanosecond range- these QDs are also expected to be less sensitive to any possible screening of the built-in electric field, be it due to the carrier injection regime (low to high) or to surrounding charges.

In this work, we investigate the distribution of radiative lifetime that occurs within a single GaN/AIN QD layer because of the dot size dispersion. We evidence that, beyond the variations stemming from the changes in dot height that give rise to an increasing weight of the QCSE, the fluctuations in their lateral size [21] explain the reduced variation in the radiative lifetime, within a factor about two, for dots whose emission energy covers a spectral range of $\sim 600 \mathrm{meV}$.

\section{SAMPLE DESCRIPTION AND EXPERIMENTAL SET-UP}

The sample consists of a single plane of GaN/AlN QDs deposited on a $c$-plane sapphire substrate by ammoniasource molecular beam epitaxy (MBE) in the StranskiKrastanov growth mode. The template is composed of a commercial $1 \mu \mathrm{m}$ thick AlN buffer layer (DOWA Electronics) grown by metal-organic vapor phase epitaxy and a $100 \mathrm{~nm}$ thick AlN layer deposited by MBE. The GaN QDs were grown under constant low ammonia flux $\left(\mathrm{NH}_{3}\right.$ beam equivalent pressure of $3 \cdot 10^{-7}$ Torr) at $750^{\circ} \mathrm{C}$ and 4.5 monolayer (ML) GaN coverage. After forming the QDs the Ga flux was stopped and the dots were capped with a $55 \mathrm{~nm}$ thick AlN barrier. The dot density under such growth conditions is in the $10^{10}-10^{11} \mathrm{~cm}^{-2}$ range [15].

Time-resolved photoluminescence (TRPL) measurements were performed at low temperature $(T=10 \mathrm{~K})$. The excitation consists in the fourth harmonic of a pulsed ytterbium laser (Tangerine model from Amplitude) emitting at $257 \mathrm{~nm}(4.82 \mathrm{eV})$ with a pulse duration of $270 \mathrm{fs}$ and a repetition rate of $50 \mathrm{kHz}$. The excitation energy is below the AlN bandgap. Moreover, the wetting layer shows a thickness between 2 and 3 MLs. As observed in a similar sample, the steady-state photoluminescence (PL) of the wetting layer occurs at energies distributed between 4.5 and $5.0 \mathrm{eV}$. We therefore assume that e-h pairs are mostly created in the wetting layer even if the excited discrete states of the QDs likely play a role in the photogeneration process.
The low repetition rate also ensured that the dots relax to their ground state between two successive excitation pulses. The energy per pulse is $0.2 \mathrm{~nJ}$ and the diameter of the excitation spot focused on the sample was about $0.4 \mathrm{~mm}$, giving an incident photon flux of $2 \cdot 10^{11}$ photons $\cdot \mathrm{cm}^{-2}$. The TRPL signal was sent into a $25 \mathrm{~cm}$-focal length spectrometer with a 150 grooves $/ \mathrm{mm}$ grating and dispersed onto the photocathode of a Hamamatsu streak camera with a spectral resolution of $0.3 \mathrm{~nm}$ and a time resolution close to $10 \mathrm{ps}$.

\section{RESULTS}

Figures 1(a) and 1(b) present the streak camera image of the QD emission recorded at low temperature $(T=10 \mathrm{~K})$ and the corresponding time-integrated PL spectrum, respectively. The PL spectrum consists of a broad band modulated by fringes due to Fabry-Perot interference occurring within the epilayer stack (see Appendix A). Because of the small size of the GaN QDs, the quantum confinement increases the recombination energy far above the GaN bandgap. A comparison with a sample where the emission of GaN QDs with different heights was investigated [22] indicates that the thickness of those QDs would range between 6 and 8 MLs. Figure 2 presents normalized PL spectra recorded at different delay times after excitation. The emission, which is centered around $3.8 \mathrm{eV}$ and has a full width at half maximum (FWHM) of $\sim 500 \mathrm{meV}$, exhibits a continuous redshift as a function of time.

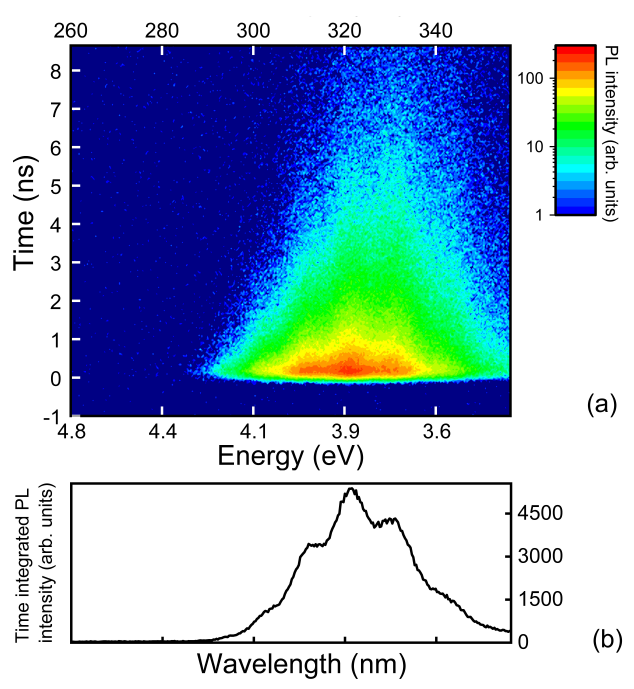

FIG. 1: (a) Streak camera image of GaN/AlN QDs recorded at $T=10 \mathrm{~K}$ using a $4.82 \mathrm{eV}$ pulsed laser for an incident photon flux of $2 \cdot 10^{11}$ photons $\cdot \mathrm{cm}^{-2}$, (b) corresponding time-integrated PL spectrum. 


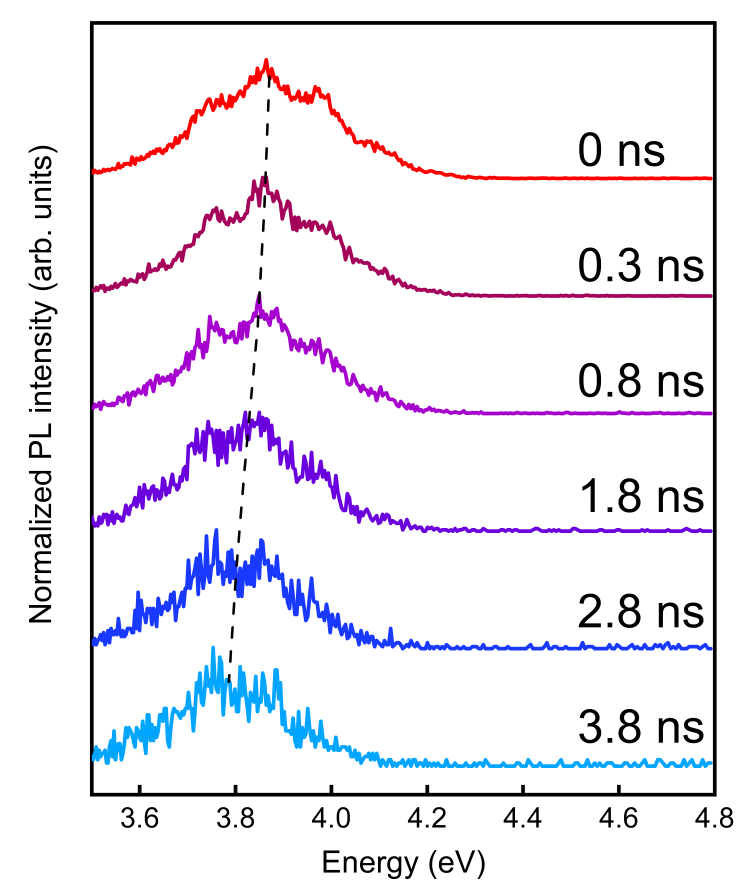

FIG. 2: Low temperature $(T=10 \mathrm{~K})$ normalized PL spectra of a GaN/AIN QD ensemble at different delay times after pulsed laser excitation. The dashed line represents the energy position of the PL maximum, $E_{\max }(t)$, obtained from Gaussian fits of the spectra.

\section{A. Mechanisms behind the redshift of the PL spectra}

Both the quantum confinement and the QCSE depend on the QD height $[19,23]$. Due to the size-dispersion of the dots, PL emission experiences an inhomogeneous broadening that we assumed to be Gaussian. This is justified by the quasi-linear dependence of the PL emission energy with the QD height as we will show in our calculations (cf. Sec. III C). An energy shift is observed between $t=0$ and $t=3.8 \mathrm{~ns}(\sim 90 \mathrm{meV})$ that is much smaller than the FWHM of the PL band $(\sim 500 \mathrm{meV})$. Two phenomena can contribute to this shift. First, because of the QCSE, the spectral contributions from dots with different heights will display different dynamics. Thus, at long time delay, the contribution from the low energy side of the PL band is enhanced with respect to the high energy emission that decays faster. The larger QDs, which emit at lower photon energies, show indeed a higher QCSE that increases the e-h spatial separation and, consequently, their radiative lifetime. Another explanation could be a possible screening of the built-in electric field: The initial e-h pair population modifies the QD band profile, inducing a blueshift of the PL emission at short time delay that will disappear with the recombination of this population [24].

Unfortunately, both effects have the same physical ori- gin and are always present and interdependent, leading to a complex PL dynamics that is challenging to interpret. However, in the limit of low carrier injection, i.e., for less than one e-h pair per dot, the screening of the electric field becomes negligible. For these reasons, we performed our experiment at low excitation intensity. Taking into account the above-mentioned photon flux and the absorption of the sample $(\simeq 3.5 \%$, measured at room temperature on a commercial UV-Vis-NIR Perkin-Elmer Lambda 950 spectrophotometer), we estimate that, in the limit where each absorbed photon creates one e-h pair, their maximum surface density would be $7 \cdot 10^{9} \mathrm{~cm}^{-2}$. Compared to the surface density of QDs given above of $10^{10}$ $10^{11} \mathrm{~cm}^{-2}$, it is reasonable to assume that there is no more than one e-h pair per dot knowing that e-h pairs will most likely not all end up in a QD (capture efficiency $<100 \%$ ). Hence, screening effects are expected to be negligible in the present set of experiments. This is also supported by modeling of the emission as demonstrated hereafter.

\section{B. Time evolution of the PL spectra}

In order to study the PL dynamics as a function of $\mathrm{QD}$ height, we have extracted several series of time-profiles integrated on spectral slices within the emission spectrum. If, as discussed above, there is no screening effect, the PL transients should exhibit an exponential decay for sufficiently narrow spectral slices. Obviously, this is not the case as can be seen in Fig. 3 that displays a few typical decay profiles spectrally integrated over $5 \mathrm{~nm}$ wide regions together with the corresponding fitting curves. The fitting function is the sum of three contributions: two exponential decays, together with a constant background. The latter represents a very long recombination process that is not resolved in our experiments. It will be discussed later on. For the first two contributions, at each spectral position, the fast component does not depend on the photon energy while the slower one follows the energy dependence expected in the context of the QCSE. In the following, we denote $\tau_{L}$ and $\tau_{S}$ the time constants related to the slow (L: long delays) and fast (S: short delays) components, respectively.

\section{1. $P L$ dynamics at short and very long delays}

The fact that, before reaching a plateau, the dynamics is bi-exponential indicates that we are dealing with two independent relaxation channels. Concerning $\tau_{S}$, its value is close to $0.3 \mathrm{~ns}$. Being independent of the photon energy (and therefore of the dot size), it does not contribute to the spectral shift described in the previous paragraph. Since $\tau_{S}$ is not affected by the dot height, it leads us to assume that it is related to a non-radiative process. Unfortunately, our experimental data do not enable us to identify in an unambiguous manner the nature 


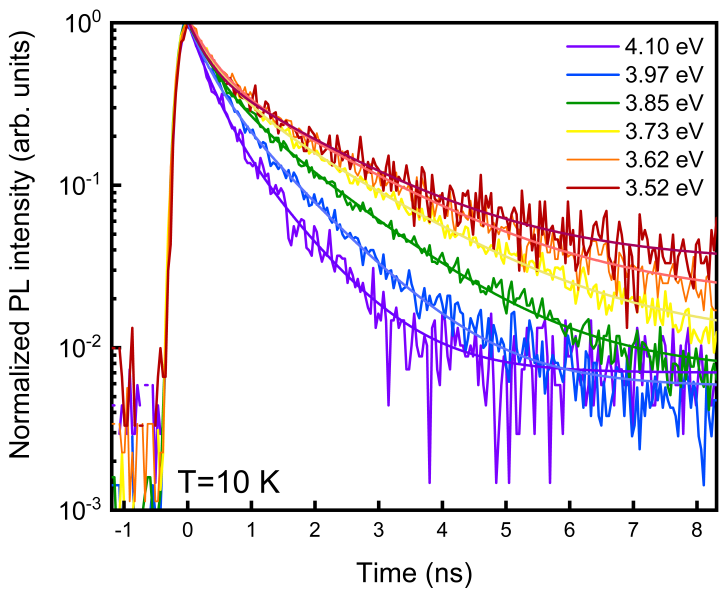

FIG. 3: Low temperature $(T=10 \mathrm{~K}) \mathrm{PL}$ decays of GaN/AlN QDs depending on the emission energy corresponding to different heights of dots.

of the electronic states that constitute the ending step of this transfer. However, as this short decay is not usually seen in experiments performed on non-unintentionally doped high quality GaN/AlGaN quantum wells (QWs) (see for exemple [25]), we postulate that these features are specific to QDs. Processes involving the trapping of a single carrier outside the dots, like in colloidal nanocrystals (see for example [26]), are very unlikely, especially when taking into account the large difference in bandgap energy between GaN and AlN. Indeed, it would imply bringing an energy of several hundreds of meV to ionize the QDs, which can be excluded at $10 \mathrm{~K}$. As less than one e- $\mathrm{h}$ pair is created within each dot, scattering events between two such pairs giving rise to an ionization are also unlikely. Moreover, such processes where a single carrier remains within the dot are known to favor nonradiative recombination like Auger processes that are not observed here. Instead, we could have either an extrinsic process, that involves for example the trapping of a carrier at a point defect within the QD or at the interface with the barrier material, or an intrinsic process, like the relaxation toward the fundamental state of the QD that would be a dark level with a different spin configuration [11].

We can now focus on the very long component that shows up as a constant background in our experiments. It could be tentatively explained by the reverse process where the e-h pairs that have experienced a relaxation toward a dark state will slowly flip back to their bright radiative state.

\section{2. $P L$ dynamics at intermediate delays}

Unlike the short decay characterized by $\tau_{S}, \tau_{L}$ shows an evolution with the photon energy that is characteristic of a height-induced variation of the radiative lifetime of a QD that undergoes the QCSE. Indeed, the experiments being performed at $10 \mathrm{~K}$, the decay that we measure is purely radiative. In Fig. 4, we have plotted the corresponding recombination rate $\gamma(E)=\left(\tau_{L}(E)\right)^{-1}$ as a function of emission energy. Its variation can be written as a Taylor expansion to second order around $E_{0}$, the initial position of the PL maximum:

$$
\gamma(E)=\gamma_{0}+\gamma_{1}\left(E-E_{0}\right)+\frac{1}{2} \gamma_{2}\left(E-E_{0}\right)^{2}
$$

$$
\text { with } \gamma_{1}=\left.\frac{d \gamma}{d E}\right|_{E_{0}} \text { and } \gamma_{2}=\left.\frac{d^{2} \gamma}{d E^{2}}\right|_{E_{0}} \text {. }
$$

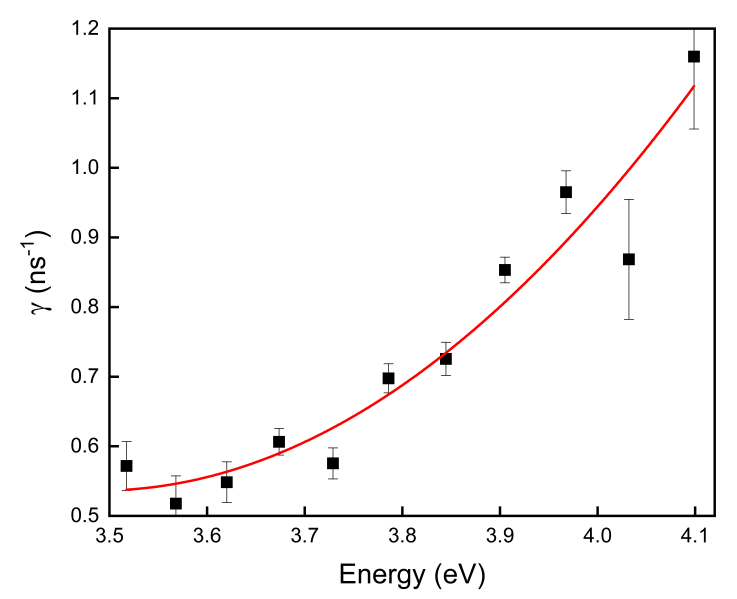

FIG. 4: Evolution of the experimental radiative rate $\gamma(E)$ deduced from the PL decays recorded at $T=10 \mathrm{~K}$ as a function of QD emission energy (black squares) and its fit using a second order polynomial (red continuous line).

The best agreement is obtained for

$$
\begin{aligned}
E_{0} & =3.869 \mathrm{eV} \\
\gamma_{0} & =0.76 \pm 0.02 \mathrm{~ns}^{-1} \\
\gamma_{1} & =1.2 \pm 0.1 \mathrm{~ns}^{-1} \mathrm{eV}^{-1} \\
\gamma_{2} & =3.1 \pm 1.3 \mathrm{~ns}^{-1} \mathrm{eV}^{-2}
\end{aligned}
$$

These values show large uncertainties, mainly because fitting the dynamics at different energies can lead to approximate values of $\gamma$ in the spectral region where the signal to noise ratio of the PL is poor. We thus used another method that allows a better precision especially on the second derivative: We perform an analysis by fitting the entire spectrum recorded at different delay times 
(Fig. 2) in order to provide a better averaged value of $\gamma(E)$.

Unsurprisingly, the spectra displayed in Fig. 2 fit nicely with a Gaussian lineshape after removing the oscillations due to the Fabry-Perot effect (see Appendix A). Indeed, the homogeneous linewidth is negligible with regard to the inhomogeneous broadening that gives rise to the observed linewidth due to the size distribution of the QDs. If screening effects are negligible, the relation between the dot height and the emission energy is expected to be a bijection. The radiative recombination probability is independent of time and the dynamics for one given height of quantum dots is exponential. Then, considering an ensemble of QDs with heights following a normal distribution, the temporal evolution of the corresponding PL intensity spectrum $I_{P L}(E, t)$ can be written:

$$
I_{P L}(E, t) \propto e^{-\frac{\left(E-E_{0}\right)^{2}}{\Gamma_{i n h}^{2}}} \cdot e^{-\gamma(E) t}
$$

where $\Gamma_{i n h}$ is the inhomogeneous broadening, $E_{0}$ is the energy of the PL maximum at zero delay, and, as above, $\gamma(E)=1 / \tau_{L}(E)$ is an implicit function describing the energy dependence of the radiative lifetime. As long as $\gamma(E)$ contains only linear and quadratic terms in $E$, the spectral shape will remain Gaussian at any time, with simply a width and a central value that change together with the maximum intensity.

The first derivative of Eq. (6) with respect to energy cancels at the maximum of the Gaussian curve, as detailed in Appendix B:

$$
E_{\max }(t)=E_{0}-\frac{\Gamma_{i n h}^{2} \gamma_{1} \cdot t}{2+\Gamma_{i n h}^{2} \gamma_{2} \cdot t} .
$$

To first order, the time evolution of the PL maximum position is thus a linear function of time. The FWHM is given by the second derivative of Eq. (6):

$$
\Delta E(t)=\frac{2 \Gamma_{i n h}}{\sqrt{2+\Gamma_{i n h}^{2} \gamma_{2} \cdot t}} .
$$

The last Gaussian parameter is the intensity:

$$
I_{P L}\left(E_{\text {max }}, t\right) \propto \exp \left(-\gamma_{0} \cdot t+\frac{1}{2}\left(\frac{\Gamma_{i n h}^{2} \gamma_{1}^{2}}{2+\Gamma_{i n h}^{2} \gamma_{2} t}\right) \cdot t^{2}\right)
$$

Using this analytic approach, the temporal behavior of the PL lineshape provides information about a possible screening of the electric field.

The time evolution of the PL lineshape parameters as well as the corresponding fits are plotted in Fig. 5, while the intensity time evolution is shown in Fig. 6. For the latter, we have to take into account the fast component decay $\tau_{S}=0.3 \mathrm{~ns}$. Therefore, we performed the fit (red curve in Fig. 6) for times longer than 0.6 ns. The experimental data are nicely described by the above-mentioned expression demonstrating that the temporal evolution of the PL line is mainly due to the variation in the radiative lifetime within the QD distribution. The fitting procedure gives:

$$
\begin{aligned}
\Gamma_{i n h} & =0.242 \pm 0.001 \mathrm{eV}, \\
\gamma_{0} & =0.77 \pm 0.02 \mathrm{~ns}^{-1}, \\
\gamma_{1} & =1.05 \pm 0.02 \mathrm{~ns}^{-1} \mathrm{eV}^{-1}, \\
\gamma_{2} & =3.08 \pm 0.36 \mathrm{~ns}^{-1} \mathrm{eV}^{-2} .
\end{aligned}
$$

As expected, this method leads to an improved precision regarding the numerical coefficients considered in the Taylor expansion of $\gamma(E)$.

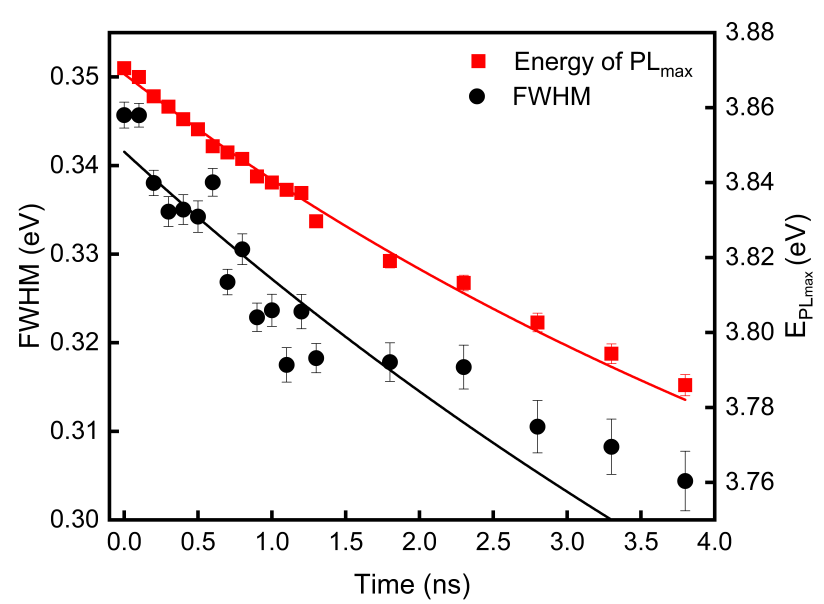

FIG. 5: Time evolution of both the center $E_{\max }(t)$ and the FWHM of the Gaussian function $\Delta E(t)$ modeling the low temperature PL spectra of GaN/AlN QDs recorded at different delay times.

\section{Dependence of the radiative lifetime on the dot height}

The coefficient $\gamma_{1}$ in the Taylor expansion of $\gamma(E)$ is positive, which reflects the increase in the recombination probability when decreasing the dot height due to the smaller weight of the QCSE. Hence, $\Delta E(t)$ is a monotonous decreasing function of time. The longer the delay time, the narrower the distribution of populated QDs because the larger radiative recombination rate of small QDs is such that only the larger dots emitting at lower photon energies will contribute to the PL spectra, thus leading to the observed decrease in the FWHM.

The time constants extracted by means of the fitting procedure are summarized in Fig. 7 together with literature data related to samples containing different heights 


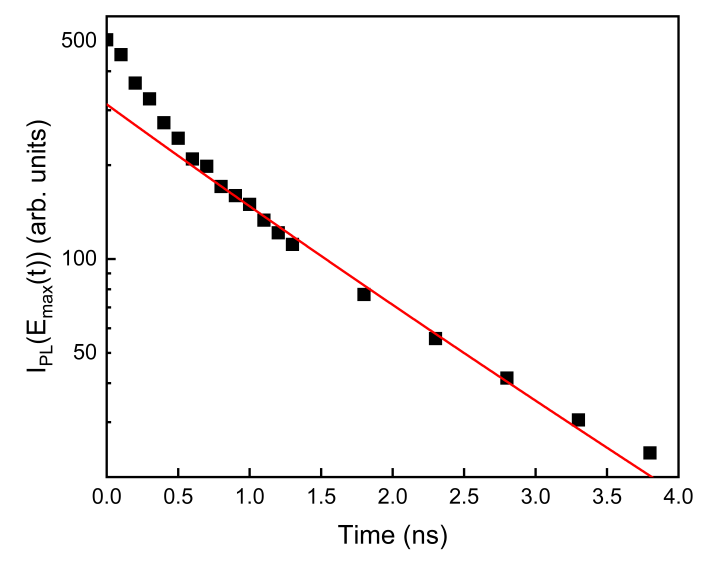

FIG. 6: Time evolution of the PL intensity at $E_{\max }(t)$.

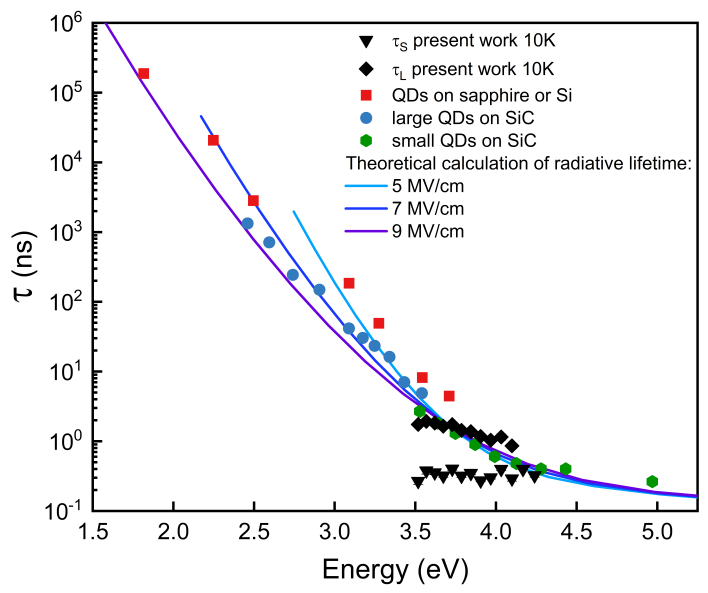

FIG. 7: Radiative lifetime $\tau_{L}$ of GaN/AlN QDs as a function of emission energy: black diamonds (present work), red squares (Ref. [17]), blue circles and green hexagons (Ref. [20]). Black down triangles correspond to the fast time constant $\tau_{S}$ (present work) ascribed to a nonradiative relaxation process. The continuous lines are results of calculations of $\tau_{\text {rad }}$ based on a $2 \mathrm{D}$ envelope-function modeling for different values of the built-in electric field.

of GaN/AlN QDs. Our experimental data complete nicely the set of data from references $[17,20]$. In order to estimate the emission energy and the radiative lifetime of e-h pairs in GaN/AlN QDs, the envelopefunction equation was solved numerically for both electrons and holes, using a finite difference method. The confinement within the layer plane, i.e., perpendicular to the growth direction is, in a first step, neglected as the built-in electric field has the most important impact on the optical properties of III-nitride based nanostructures grown along the $c$-axis. Thus, the QDs are first mod-

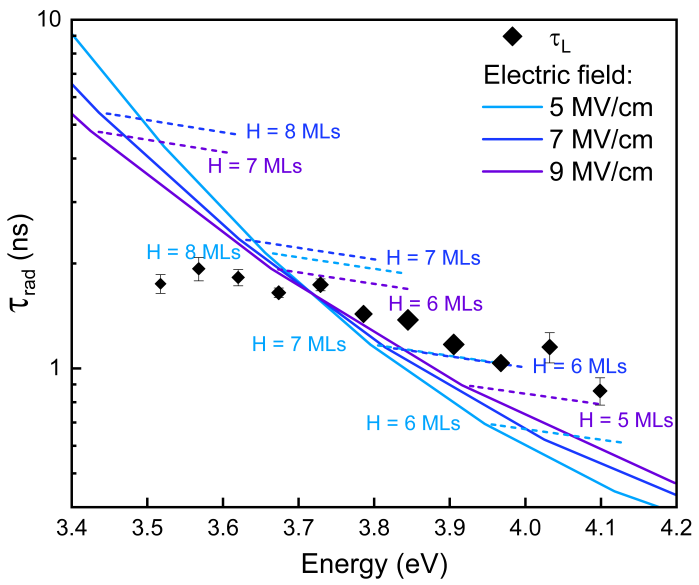

FIG. 8: Radiative lifetime $\tau_{L}$ of GaN/AlN QDs as a function of emission energy. The continuous lines are issued from $2 \mathrm{D}-\mathrm{QW}$ calculations of $\tau_{\text {rad }}$ for different values of the built-in electric field assuming a continuous variation in the dot height. The dotted lines show the effect of the QD lateral size with radii ranging between 3 and $20 \mathrm{~nm}$. Each curve corresponds to a different QD height expressed in MLs. The size of the black diamonds is proportional to the PL intensity, which reflects the weight of the corresponding dot height within the QD distribution.

eled as two-dimensional (2D) QWs subjected to a static electric field along the growth direction, the carriers being free to propagate in the well plane. We took into account the blueshift of the GaN bandgap to $3.71 \mathrm{eV}$ at low temperature $(T=10 \mathrm{~K})$ due to the strain originating from pseudomorphic growth on the AlN layer. The radiative lifetime $\tau_{\text {rad }}$ is then given by the expression: $A \cdot(\hbar \omega)^{-3}\left|J_{e h}\right|^{-2}[27]$ where $\hbar \omega$ is the photon energy, $J_{e h}$ is the overlap integral of electron and hole envelope-functions and $A$ is an adjustable parameter. The resulting function $\tau_{\text {rad }}(\hbar \omega)$ is plotted as a continuous line in Fig. 7 for different values of the electric field $F$ and $A=20 \mathrm{~ns} \cdot \mathrm{eV}^{-1}$. Such a simple calculation provides curves that qualitatively reproduce the experimental variation in the radiative lifetime over six orders of magnitude for values of the electric field ranging between $F=5 \mathrm{MV} / \mathrm{cm}$ and $F=9 \mathrm{MV} / \mathrm{cm}$, the best agreement being obtained for a mean built-in electric field value $F=7 \mathrm{MV} / \mathrm{cm}$. The mean value of our experimental data agrees well with compiled data from the literature showing the variation of the radiative lifetime for a large range of dot heights.

In order to be more quantitative, let us note that the QD ensemble in our sample exhibits a large inhomogeneous broadening. To illustrate this aspect, we consider Fig. 8, which shows that the variation in the radiative lifetime does not exactly match the curves (continuous lines) that only take into account the variation of the 
QCSE due to the changes in dot height. In fact, the fluctuations occurring within the dot ensemble are likely to also originate from the lateral confinement with an increasing weight of the latter when the QD lateral size becomes comparable to the exciton Bohr radius. Indeed, the 2D QW model works better for the largest dots for which variations in the oscillator strength are essentially due to changes in the e-h overlap along the growth axis. For small dots, the in-plane localization of the envelopefunctions is no longer negligible. To estimate this effect on the energy and the lifetime of an e-h pair, we performed a simple calculation based on the variational method (see Appendix C): the in-plane confinement potential is supposed to be step-like with a cylindrical symmetry. The in-plane envelope function of the carriers is considered to be a Gaussian centered in the cylindrical dot and for which the spatial extent is the variational parameter. This method is known to give reasonable qualitative results in spite of its simplicity [28]. For a given QD height, a variation in the localization radius has little influence on the lateral overlap while it strongly affects the confinement energy. As a consequence, the variation in the lifetime versus energy becomes less pronounced. This is illustrated in Fig. 8 that shows as dashed lines the effect of the variation in the lateral dot size on the transition energy and the radiative lifetime for various dot heights. We thus see that the slope of the dashed lines nicely matches the relatively flat variation in the radiative lifetime as a function of photon emission energy observed experimentally. Obviously, more realistic calculations would consider the different lateral confinement experienced by electrons and holes. First, these carriers have different effective masses and the wave function of the lighter particle, the electron, should leak into the barrier. Second, if the dot shape looks like truncated pyramids, as confirmed by high-resolution transmission electron microscopy measurements [29], the confinement potential would act differently on the two particles which are pushed away to opposite sides, the electron to the top of the pyramid and the hole in the wetting layer. Hence, both effects will change the lateral overlap of the electron and hole wave functions [30]. However, our approach shows that such a detailed description of the QD wave functions is not mandatory. The lateral confinement plays a major role by blueshifting the emission energy without changing the e-h overlap and thus the radiative lifetime.

Finally, we can interpret these data in the following way: from one dot to the other any variation in the mean radiative lifetime is primarily governed by a change in the dot height that involves the QCSE as already demonstrated in many publications (see, e.g., Fig. 7 that displays literature data). However, the present analysis shows that, within a given QD sample, changes in the QD emission energy by several hundreds of $\mathrm{meV}$ accompanied with only slight changes in the radiative lifetime can be understood through fluctuations in the lateral dot dimensions within a given QD subset corresponding to a well- defined height. In this respect, we recall that the identification of GaN/AlN QD subsets with a well-defined height within the same sample was already reported for dots exhibiting a variation in the biexciton binding energy that was correlated to the exciton energy and the dot radius within a QD subset with a given height [22]. Let us note that for QDs emitting around 3.5-4.1 eV, as those studied here, the effect of the built-in electric field is less important than in higher dots where the QCSE has more dramatic effects on the emission energy and the oscillator strength and for which only a mean radius has to be considered. Thus, in dots emitting above the bulk GaN bandgap, which are of prime interest for single photon emission [10,11], the weaker weight of the QCSE enables to reveal otherwise hidden effects originating from changes in the e-h lateral confinement, be it what governs variations in the biexciton binding energy [22] or a relative insensitivity of the radiative lifetime while covering a wide spectral range in the QD emission energy (here, up to $\sim 600 \mathrm{meV}$ ).

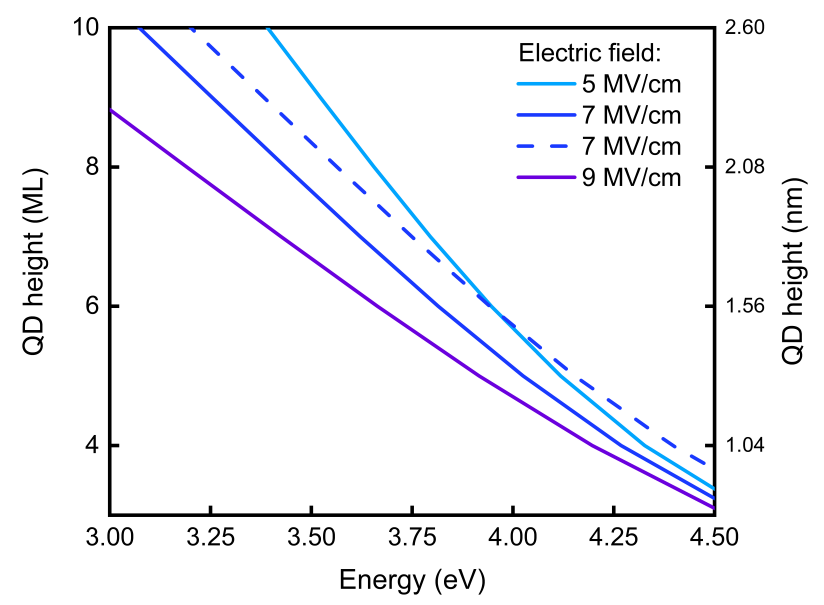

FIG. 9: Correlation between the calculated transition energy and the QD height for different values of the built-in electric field. The straight lines correspond to calculations based on a 2D QW model (no lateral confinement), while the dashed one correspond to calculations based on the variational method for small dots with a lateral radius of $3 \mathrm{~nm}$.

The reported behavior confirms that the main mechanism behind the relaxation of the e-h pair population is the radiative recombination process and that the radiative lifetime follows the expected evolution for QDs subjected to the QCSE. Moreover, our results strengthen the fact that, if care is taken to avoid any screening of the built-in electric field, the relation between the radiative lifetime and the emission energy tends to a universal trend (Fig. 7). This can be well understood within a simple model including a mean value of the built-in electric field that is the same for all GaN/AlN QD samples. 
From a practical point of view, the calculations presented in Fig. 9 allow to estimate that the QD height lies between 5 and $8 \mathrm{MLs}(1.3$ and $2.08 \mathrm{~nm})$, since $1 \mathrm{ML}$ of GaN along the $c$-axis is equal to $\sim 0.26 \mathrm{~nm}[31]$.

\section{CONCLUSION}

We have performed low temperature TRPL experiments on a single plane of GaN/AlN QDs, whose emission energy mostly lies above the bulk GaN bandgap. The PL data show a three-component dynamics: a first exponential decay with a short time-constant (0.3 ns), independent of photon energy, a second one that follows the expected evolution for the radiative lifetime with increasing QD height, and finally a very long component with a plateau-like behavior.

We attribute the fast dynamics to a nonradiative relaxation process of e-h pairs toward a dark state and the concomitant existence of a very long component, which shows up as a constant background in the PL decays, that corresponds to the reverse process where the dots come back to their original state.

Concerning the dynamics of the radiative recombination process, we have established that, in the absence of any screening of the built-in electric field, the PL spectrum of QDs with a normal height distribution presents a Gaussian lineshape with a characteristic time evolution that is easily described by an analytical model: after a pulsed excitation the position of the line maximum undergoes a redshift that varies linearly with increasing delay time and is accompanied by a decrease in the FWHM. This behavior originates from the combined effect of the quantum confinement and the QCSE, and constitutes a criterion to conclude on the possible occurrence of screening and/or many-body effects in the population dynamics within GaN/AlN QDs. Moreover, our analysis enables an improved precision concerning the determination of the energy-dependence of the QD radiative lifetime when compared to a method that involves a systematic study of the decay-profiles corresponding to different narrow PL-energy windows.

By completing previous results from other groups, our data provide new evidence that the dependence of the effective PL lifetime with emission energy follows a global trend that is an intrinsic feature of the GaN/AlN QD system where the built-in electric field acts as the most dramatic parameter. Numerical simulations, which show a good qualitative agreement over an energy range of $1 \mathrm{eV}$ and six orders of magnitude for the effective PL lifetime when assuming a built-in electric field value of $7 \mathrm{MV} / \mathrm{cm}$, enable to estimate that the dot height in our sample is distributed around a mean value of $1.6 \mathrm{~nm}(\sim 6 \mathrm{MLs})$. Remarkably, in small QDs emitting at high photon energies, around 3.5-4.1 eV, and hence for which the impact of QCSE is less pronounced, we can evidence the effects of lateral confinement on the carriers. The latter affects the wave function extent and the emission energy but only slightly the carrier overlap and the corresponding oscillator strength (equivalently the radiative lifetime).

\section{ACKNOWLEDGMENTS}

This work was funded by the French National Research Agency (ANR) through the Programme d'Investissement d'Avenir under contract ANR-17-EURE-0024 within the Investissement d'Avenir program ANR-10-IDEX-000202 .

\section{Appendix A: Removing the oscillations from PL spectra}

As shown in Fig. 10, PL spectra are modulated by fringes due to Fabry-Perot interferences occurring in the epilayer stack. We remove these oscillations by applying a Gaussian fit to time-sliced PL spectra in order to get access to the time evolution of the fitting parameters: the energy position of the PL maximum $E_{\max }$, its width and its intensity.

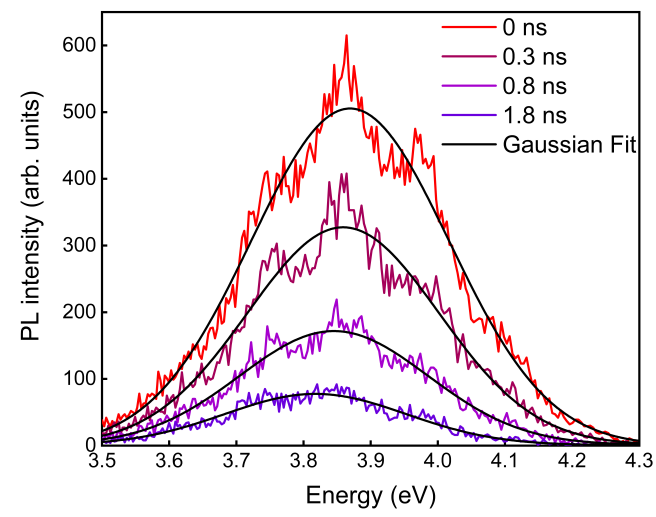

FIG. 10: Low temperature $(T=10 \mathrm{~K}) \mathrm{PL}$ spectra of a GaN/AlN QD ensemble at different delay times after the laser pulse excitation.

\section{Appendix B: Modeling of the spectro-temporal evolution of the PL spectra}

In order to obtain an analytical expression of the spectro-temporal evolution of the PL spectrum emitted by an ensemble of GaN/AlN QDs, we first make the assumption that the height distribution is normal, resulting in a Gaussian lineshape. If the density of injected e-h pairs is small enough, the recombination probability is time-independent and it exists a function $\tau(E)$ describing the variation of the radiative lifetime as a function of emission energy. The consequence is that the dynamics 
of each spectral component is exponential with a decay time $\tau(E)$.

The PL intensity can then be written:

$$
I_{P L}(E, t) \propto \exp \left[-\frac{\left(E-E_{0}\right)^{2}}{\Gamma_{i n h}^{2}}\right] \exp (-\gamma(E) t),
$$

where we have introduced the radiative relaxation rate:

$$
\gamma(E)=\frac{1}{\tau(E)} .
$$

When $\gamma$ only depends linearly and quadratically on $E$, i.e., when its Taylor expansion is stopped at the second order, the shape of $I_{P L}$ as a function of photon energy $E$ remains a Gaussian at any time. The energy position of the PL maximum $E_{\max }(t)$ and the FWHM $\Delta E(t)$ versus time are obtained by canceling the first and the second derivatives of Eq.(6) relative to the photon energy $E$, respectively. The calculation of these derivatives is straightforward and gives:

$$
\begin{aligned}
& \frac{\partial}{\partial E} I_{P L}(E, t)= \\
& \quad\left(-\frac{d \gamma}{d E} \cdot t-\frac{2\left(E-E_{0}\right)}{\Gamma_{i n h}^{2}}\right) \cdot I_{P L}(E, t),
\end{aligned}
$$

and

$$
\begin{aligned}
& \frac{\partial^{2}}{\partial E^{2}} I_{P L}(E, t)= \\
& \left(-t \cdot \frac{d^{2} \gamma}{d E^{2}}+t^{2} \cdot\left(\frac{d \gamma}{d E}\right)^{2}+\frac{4 t}{\Gamma_{i n h}^{2}}\left(\frac{d \gamma}{d E}\right)\left(E-E_{0}\right)-\right. \\
& \left.\frac{2}{\Gamma_{i n h}^{2}}+\frac{4}{\Gamma_{i n h}^{4}}\left(E-E_{0}\right)^{2}\right) \cdot I_{P L}(E, t) .
\end{aligned}
$$

\section{1. $\gamma$ is independent of $E$}

If $\gamma=\gamma_{0}$ is independent of $E$, the first derivative cancels for:

$$
E_{\max }=E_{0}
$$

and the second derivative for:

$$
E_{ \pm}=E_{0} \pm \frac{\Gamma_{i n h}}{\sqrt{2}} .
$$

Hence, the FWHM is given by:

$$
\Delta E=E_{+}-E_{-}=\sqrt{2} \Gamma_{i n h} .
$$

We recover the simple case of a Gaussian lineshape whose spectral position is independent of time and whose intensity decays with a lifetime $\tau_{0}=\gamma_{0}^{-1}$.

\section{General case: $\gamma$ is a function of $E$}

If now $\gamma$ is a quadratic function of $E$ that we can expand around $E_{0}$ :

$$
\gamma(E)=\gamma_{0}+\gamma_{1} \cdot\left(E-E_{0}\right)+\frac{1}{2} \gamma_{2} \cdot\left(E-E_{0}\right)^{2} .
$$

The first derivative of $I_{P L}$ cancels for:

$$
\begin{aligned}
E_{\text {max }}(t) & =E_{0}-\frac{\Gamma_{i n h}^{2}}{2} \frac{d \gamma}{d E} \cdot t \\
& =E_{0}-\frac{\Gamma_{i n h}^{2}}{2}\left(\gamma_{1}+\gamma_{2} \cdot\left(E_{\text {max }}(t)-E_{0}\right)\right) \cdot t, \\
E_{\text {max }}(t) & =E_{0}-\frac{\Gamma_{i n h}^{2} \gamma_{1} \cdot t}{2+\Gamma_{i n h}^{2} \gamma_{2} \cdot t} .
\end{aligned}
$$

The time evolution of the FWHM is obtained by canceling the second derivative, which gives:

$$
\begin{aligned}
E_{ \pm}(t) & =E_{\text {max }}(t) \pm \frac{\Gamma_{i n h}}{\sqrt{2}} \sqrt{1+\frac{\Gamma_{i n h}^{2}}{2} \frac{d^{2} \gamma}{d E^{2}} \cdot t} \\
& =E_{0}-\frac{\Gamma_{i n h}^{2} \gamma_{1} \cdot t \mp \Gamma_{i n h} \sqrt{2+\Gamma_{i n h}^{2} \gamma_{2} \cdot t}}{2+\Gamma_{i n h}^{2} \gamma_{2} \cdot t}
\end{aligned}
$$

$$
\Delta E(t)=E_{+}(t)-E_{-}(t)=\frac{2 \Gamma_{i n h}}{\sqrt{2+\Gamma_{i n h}^{2} \gamma_{2} \cdot t}} .
$$

The intensity is then given by the PL emission maximum at $E_{\max }(t)$ :

$$
I_{P L}\left(E_{\max }, t\right) \propto \exp \left(-\gamma_{0} \cdot t+\frac{1}{2}\left(\frac{\Gamma_{i n h}^{2} \gamma_{1}^{2}}{2+\Gamma_{i n h}^{2} \gamma_{2} t}\right) \cdot t^{2}\right)
$$

\section{Appendix C: Variational calculation of in-plane wave-functions}

A variational calculation enables to estimate the influence of an in-plane confinement potential on the energies and radiative lifetimes of QDs. We consider as a first approximation that the potential is separable in the $z$ and $(x, y)$ directions. The built-in electric field is taken into account along the vertical direction and does not play any role on the lateral confinement. The Hamiltonian of a particle with effective mass $m^{*}$ trapped in an abrupt potential $V_{0}$ with a cylindrical symmetry is given by:

$$
H=-\frac{\hbar^{2}}{2 m^{*}} \frac{1}{\rho} \frac{\partial}{\partial \rho}\left(\rho \frac{\partial}{\partial \rho}\right)+V_{0} \Theta\left(\rho-\rho_{0}\right),
$$


where $\rho$ is the radial coordinate, $\Theta$ is the Heaviside step function and $\rho_{0}$ is the spatial extension of the lateral potential. The confinement energy can be found using the variational method by minimizing:

$$
E\left(\rho_{0}, \sigma\right)=\langle\phi(\sigma)|H| \phi(\sigma)\rangle
$$

as a function of the extension parameter $\sigma$, where $\phi$ is a normalized Gaussian envelope-function:

$$
\phi(\rho)=\frac{1}{\sigma \sqrt{\pi}} \exp \left[-\left(\frac{\rho}{\sigma}\right)^{2}\right]
$$

The problem is solved analytically and gives:

$$
\sigma=\rho_{0} \sqrt{2}\left[\ln \left(\frac{2 m^{*} \rho_{0}^{2} V_{0}}{\hbar^{2}}\right)\right]^{-1 / 2},
$$

and

$$
E\left(\rho_{0}\right)=\frac{\hbar^{2}}{2 m^{*} \rho_{0}^{2}}\left[1+\ln \left(\frac{2 m^{*} \rho_{0}^{2}}{\hbar^{2}} V_{0}\right)\right] .
$$

The procedure is realized for electrons and holes with effective masses $m_{e}^{*}=0.2 m_{0}$ and $m_{h}^{*}=0.33 m_{0}$ [32], respectively, with $m_{0}$ the free electron mass, giving the confinement energies $E_{e}$ and $E_{h}$, as well as the in-plane extensions $\sigma_{e}$ and $\sigma_{h}$ of the corresponding envelopefunctions. The overlap integral can also be evaluated analytically:

$$
\int_{0}^{+\infty} \phi_{e}(\rho) \phi_{h}(\rho) 2 \pi \rho d \rho=2 \frac{\sigma_{e} \sigma_{h}}{\sigma_{e}^{2}+\sigma_{h}^{2}} .
$$

[1] P. Michler, A. Imamoglu, M. D. Mason, P. J. Carson, G. F. Strouse, and S. K. Buratto, Nature 406, 968 (2000).

[2] C. L. Salter, R. M. Stevenson, I. Farrer, C. A. Nicoll, D. A. Ritchie, and A. J. Shields, Nature 465, 594 (2010).

[3] G. Juska, V. Dimastrodonato, L. O. Mereni, A. Gocalinska, and E. Pelucchi, Nature Photonics 7, 527 (2013).

[4] T. Heindel, C. A. Kessler, M. Rau, C. Schneider, M. Fürst, F. Hargart, W. M. Schulz, M. Eichfelder, R. Roßbach, S. Nauerth, M. Lermer, H. Weier, M. Jetter, M. Kamp, S. Reitzenstein, S. Höfling, P. Michler, H. Weinfurter, and A. Forchel, New Journal of Physics 14, 083001 (2012).

[5] W. G. van der Wiel, M. Stopa, T. Kodera, T. Hatano, and S. Tarucha, New Journal of Physics 8, 28 (2006).

[6] W. Zhou and J. J. Coleman, Current Opinion in Solid State and Materials Science 20, 352 (2016).

[7] I. Robert-Philip, E. Moreau, S. Varoutsis, J. Bylander, M. Gallart, J.-M. Gérard, and I. Abram, Journal of Luminescence 102-103, 67 (2003).

[8] J. Bylander, I. Robert-Philip, and I. Abram, The European Physical Journal D 22, 295 (2003).

[9] B. Damilano, N. Grandjean, F. Semond, J. Massies, and M. Leroux, physica status solidi (b) 216, 451 (1999).

[10] M. J. Holmes, S. Kako, K. Choi, M. Arita, and Y. Arakawa, Nano Lett. 14, 982 (2014).

[11] S. Tamariz, G. Callsen, J. Stachurski, K. Shojiki, R. Butté, and N. Grandjean, ACS Photonics 7, 1515 (2020).

[12] M. J. Holmes, K. Choi, S. Kako, M. Arita, and Y. Arakawa, ACS Photonics 3, 543 (2016).

[13] D. Lagarde, A. Balocchi, H. Carrère, P. Renucci, T. Amand, X. Marie, S. Founta, and H. Mariette, Phys. Rev. B 77, 041304 (2008).

[14] B. Damilano, J. Brault, and J. Massies, Journal of Applied Physics 118, 024304 (2015).

[15] S. Tamariz, G. Callsen, and N. Grandjean, Applied
Physics Letters 114, 082101 (2019).

[16] F. Bernardini, V. Fiorentini, and D. Vanderbilt, Phys. Rev. B 56, R10024 (1997).

[17] T. Bretagnon, P. Lefebvre, P. Valvin, R. Bardoux, T. Guillet, T. Taliercio, B. Gil, N. Grandjean, F. Semond, B. Damilano, A. Dussaigne, and J. Massies, Phys. Rev. B 73, 113304 (2006).

[18] L. Zhou, D. J. Smith, M. R. McCartney, T. Xu, and T. D. Moustakas, Applied Physics Letters 99, 101905 (2011).

[19] A. D. Andreev and E. P. O'Reilly, Applied Physics Letters 79, 521 (2001).

[20] S. Kako, M. Miyamura, K. Tachibana, K. Hoshino, and Y. Arakawa, Applied Physics Letters 83, 984 (2003).

[21] M. Winkelnkemper, M. Dworzak, T. P. Bartel, A. Strittmatter, A. Hoffmann, and D. Bimberg, Physica Status Solidi (b) 245, 2766 (2008).

[22] D. Simeonov, A. Dussaigne, R. Butté, and N. Grandjean, Phys. Rev. B 77, 075306 (2008).

[23] D. P. Williams, A. D. Andreev, and E. P. O'Reilly, Phys. Rev. B 73, 241301(R) (2006).

[24] M. Henini, Handbook of self assembled semiconductor nanostructures for novel devices in photonics and electronics (Elsevier, Amsterdam, 2008) pp. 236-260.

[25] F. Stokker-Cheregi, A. Vinattieri, E. Feltin, D. Simeonov, J.-F. Carlin, R. Butté, N. Grandjean, F. Sacconi, M. Povolotskyi, A. Di Carlo, and M. Gurioli, Phys. Rev. B 79, 245316 (2009).

[26] F. M. Gómez-Campos and M. Califano, Hole surface trapping in cdse nanocrystals: Dynamics, rate fluctuations, and implications for blinking, Nano Letters 12, 4508 (2012).

[27] A. Thränhardt, C. Ell, G. Khitrova, and H. M. Gibbs, Phys. Rev. B 65, 035327 (2002).

[28] J.-Y. Marzin and G. Bastard, Solid State Communications 92, 437 (1994).

[29] M. Arlery, J.-L. Rouvière, F. Widmann, B. Daudin, 
G. Feuillet, and H. Mariette, Applied Physics Letters 74, 3287 (1999).

[30] R. Butté and N. Grandjean, Effects of polarization in optoelectronic quantum structures, in Polarization Effects in Semiconductors: From Ab Initio Theory to Device Applications, edited by C. Wood and D. Jena (Springer US,
New York, 2008) pp. 467-511.

[31] I. Vurgaftman and J. R. Meyer, Journal of Applied Physics 94, 3675 (2003).

[32] P. Rinke, M. Winkelnkemper, A. Qteish, D. Bimberg, J. Neugebauer, and M. Scheffler, Phys. Rev. B 77, 075202 (2008). 